\title{
Correction to: Physiologically based pharmacokinetic modelling to predict the clinical effect of CYP3A inhibitors/inducers on esaxerenone pharmacokinetics in healthy subjects and subjects with hepatic impairment
}

Akiko Watanabe $^{1}$ (1) $\cdot$ Tomoko Ishizuka $^{2} \cdot$ Makiko Yamada $^{2} \cdot$ Yoshiyuki Igawa $^{1} \cdot$ Takako Shimizu $^{1} \cdot$ Hitoshi Ishizuka $^{1}$

Published online: 8 September 2021

๑) Springer-Verlag GmbH Germany, part of Springer Nature 2021

\section{Correction to: European Journal of Clinical Pharmacology https://doi.org/10.1007/s00228-021-03194-x}

In Eq. 3, the -1 should not be in superscript.

The Original article has been corrected.

Publisher's Note Springer Nature remains neutral with regard to jurisdictional claims in published maps and institutional affiliations.

The original article can be found online at https://doi.org/10.1007/ s00228-021-03194-x.

Akiko Watanabe

watanabe.akiko.mi@daiichisankyo.co.jp

1 Quantitative Clinical Pharmacology Department, Daiichi, Sankyo Co., Ltd., Tokyo, Japan

2 Drug Metabolism and Pharmacokinetics Research, Laboratories, Daiichi Sankyo Co., Ltd., Tokyo, Japan 